Research Article

\title{
Chemical Functionalization Effects on Cubane-Based Chain Electronic Transport
}

\author{
Konstantin P. Katin ${ }^{1,2}$ and Mikhail M. Maslov ${ }^{1,2}$ \\ ${ }^{1}$ Department of Condensed Matter Physics, National Research Nuclear University "MEPhI", Kashirskoe Shosse 31, \\ Moscow 115409, Russia \\ ${ }^{2}$ Laboratory of Computational Design of Nanostructures, Nanodevices and Nanotechnologies, Research Institute for \\ the Development of Scientific and Educational Potential of Youth, Aviatorov Street 14/55, Moscow 119620, Russia \\ Correspondence should be addressed to Mikhail M. Maslov; mike.maslov@gmail.com
}

Received 5 May 2015; Revised 20 July 2015; Accepted 28 July 2015

Academic Editor: Sergio E. Ulloa

Copyright ( $\odot 2015$ K. P. Katin and M. M. Maslov. This is an open access article distributed under the Creative Commons Attribution License, which permits unrestricted use, distribution, and reproduction in any medium, provided the original work is properly cited.

\begin{abstract}
We report electronic structure calculations in chemically functionalized linear cubane-based chains. The effects of covalent chemical attachments on chain transport properties are examined with nonorthogonal tight-binding model (NTBM) considering LandauerBüttiker formalism. The covalent bonding of even a single-type functional group is shown to considerably alter the conductance of the chain. For similar radical doping density, electronic characteristics are found to range from insulator to narrow-gap semiconductor depending on the nature of the covalent bonding. Therefore it has become possible to tune electronic properties of the cubane-based one-dimensional oligomers by the functionalization for nanoelectronic applications.
\end{abstract}

\section{Introduction}

Hydrocarbon cubane $\mathrm{C}_{8} \mathrm{H}_{8}$ synthesized in 1964 [1] remains the focus of modern research because of its unusual geometry, energy, and electronic properties. In cubane molecule the valence angles between the $\mathrm{C}-\mathrm{C}$ covalent bonds are $90^{\circ}$, distinct from the value $109.5^{\circ}$, typical of $s p^{3}$-hybridized carbon compounds. Nevertheless, despite the strained carbon cage (strain energy is about $670 \mathrm{~kJ} / \mathrm{mol}$ [2]) cubane molecules demonstrate surprisingly high kinetic stability [3] and can form various derivatives, in which one or more hydrogen atoms are replaced by functional groups or radicals. For example, cubane-based systems with methyl groups [4], propyl groups [4], carboxyl groups [5], and nitrogroups [68] are successfully synthesized. Moreover, dehydrogenated cubanes can form oligomers through covalent bonding. The cubane dimer (cubylcubane) [9], linear chains constructed from elementary cubyl fragments [5], and cubane-based solids $[10,11]$ are intensively studied as well. Although a number of cubane-based oligomers remain hypothetical and are not obtained experimentally, they are examined theoretically, including numerical simulation. Both $a b$ initio $[12,13]$ and semiempirical $[14,15]$ calculations predict thermodynamic and kinetic stabilities of one- and two-dimensional nanostructures built from cubylene units (chains, rings, and networks). These systems are of particular interest because of their remarkable electronic properties [16-19]. For example, polycubane chains linked by various small molecules ("molecular bridges") demonstrate a variety of electronic characteristics: from strong insulators to metals [17]. Thus, it becomes possible to design novel advanced materials with adjustable electronic structure. Recently reported charge transfer between donor and acceptor groups in cubanebased one-dimensional systems makes these nanostructures perspective materials to be used in pull-push devices [18].

Linear chains built from cubylene units [5] are the simplest members of the cubane-based structures family. $A b$ initio calculations [13] predict that the sufficiently long chain is a strong insulator with the energy gap equal to $4.59 \mathrm{eV}$, but this value can be reduced through doping the initial system by donor/acceptor functional groups $[13,17]$. Nevertheless, the mechanism of the effect of chemical functionalization on the energy gap of cubane-based nanostructures is still not clear.

In this paper we present electronic structure calculations for the linear chains built from substituted cubylene units. In each unit one or more hydrogen atoms are replaced by functional groups or radicals. We restrict ourselves to $\mathrm{H}-\mathrm{C}-\mathrm{N}-\mathrm{O}$ 
chemical groups with various electronegativities, for which corresponding substituted cubanes are already synthesized or can be easily prepared in the future. The main purpose of this work is to demonstrate the electronic properties of substituted cubane-based chains dependence of the chemical groups attached and to calculate their conductance.

\section{Materials and Methods}

In our study we use the nonorthogonal tight-binding model originally developed for $\mathrm{H}-\mathrm{C}-\mathrm{N}-\mathrm{O}$ systems $[20,21]$ as well as high-level density functional theory (DFT). Density functional theory with B3LYP $[22,23]$ and PBE [24] functionals with the electron basis sets of $6-311 \mathrm{G} / 6-311 \mathrm{G}(\mathrm{d}, \mathrm{p})$ and $6-31+G(d, p)[25]$ was used. All DFT calculations were performed using the GAMESS program package [26]. The parameters' fitting of tight-binding model is based on the criterion of the best correspondence between the computed and experimental (not derived from $a b$ initio calculations) values of binding energies, bond lengths, and valence angles of several selected small $\mathrm{H}_{k} \mathrm{C}_{l} \mathrm{~N}_{m} \mathrm{O}_{n}$ molecules (for parameterization details see $[20,27])$. To validate the model developed, we computed the binding energies, bond lengths, and valence angles for various $\mathrm{H}-\mathrm{C}-\mathrm{N}-\mathrm{O}$ compounds not used in the fitting procedure and compared them with the experimental data [28]. The resulting tight-binding potential has a good transferability and is well suited to modeling various $\mathrm{H}_{k} \mathrm{C}_{l} \mathrm{~N}_{m} \mathrm{O}_{n}$ molecules [27] from small clusters to large systems as graphene, diamond, different peptides, and so forth. Such tight-binding approach is commonly used for electronic structure calculations [29-32], including HOMOLUMO gaps, density of states, and transmission coefficients.

The samples studied are constructed from eleven functionalized cubylene fragments. Each structure is obtained from pristine chain (see Figure 1(a)) by replacing a hydrogen atom with chemical functional group or radical: $\mathrm{CH}_{3}, \mathrm{OH}$, $\mathrm{NH}_{2}, \mathrm{CN}, \mathrm{COOH}$ (Figure 1(b)), $\mathrm{NO}_{2}$, and $\mathrm{NO}$ (Figure 1(c)). The choice of these functional groups will be clear from the analysis below. So, the total number of oligomers investigated is equal to eight.

To obtain the equilibrium structures of the cubane-based oligomers we applied the method of structural relaxation by using the same nonorthogonal tight-binding model (NTBM) $[20,21,27]$. The corresponding initial configuration relaxed to a state with the local or global energy minimum under the influence of intramolecular forces only. First of all, the forces acting on all atoms were calculated using the HellmannFeynman theorem. Next, atoms were shifted in the direction of the forces obtained proportional to the corresponding forces. Then the relaxation step was repeated. The whole structure is relaxed up to residual atomic forces smaller than $10^{-4} \mathrm{eV} / \AA$. Note that for all cubane-based chains studied we also calculated the frequency spectra in the framework of the same level of theory after the geometry optimization. For each metastable configuration, the presence of local minima of energy was confirmed by the reality of all frequencies. We have verified on the smaller oligomers that optimizing the structures in this way leads to minor changes in the energy and electronic characteristics (e.g., binding energies

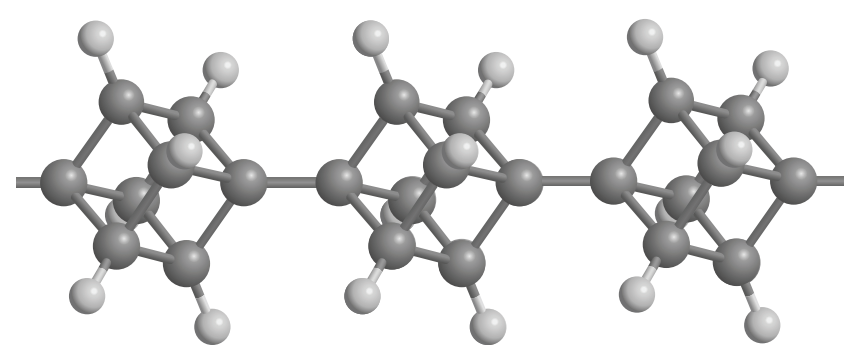

(a)

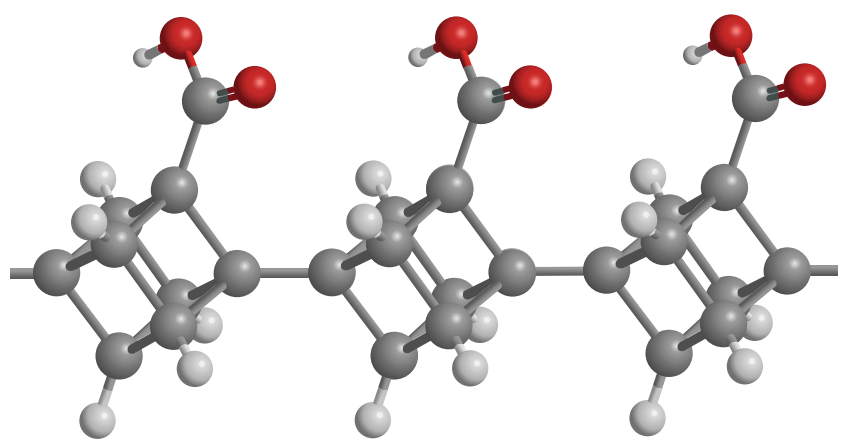

(b)

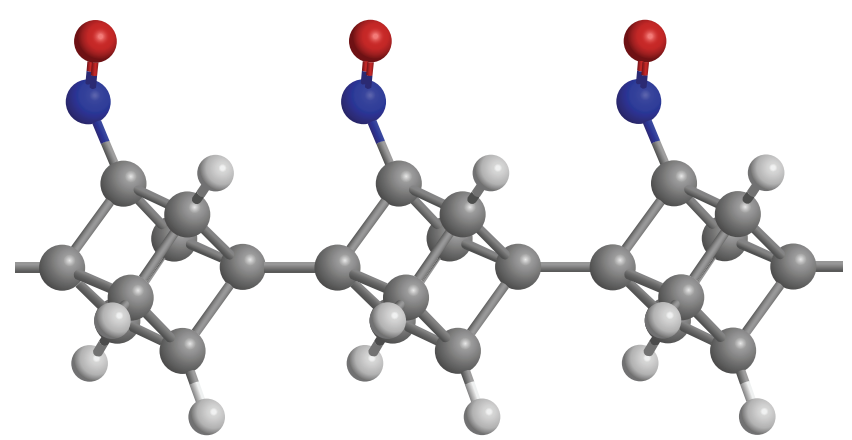

(c)

FIGURE 1: Fragments of the chemically functionalized cubane-based chains studied: (a) pristine chain; (b) $\mathrm{COOH}$-functionalized chain; (c) NO-functionalized chain.

and HOMO-LUMO gaps) as compared to high-level DFT relaxation. Results obtained for HOMO-LUMO gaps for cubane-based oligomers constructed from two and four fragments (dimers and tetramers) are presented in Table 1. From Table 1 one can see that the accuracy of the NTBM is in good agreement with the DFT approach (except, maybe, $\mathrm{CN}$-functionalization).

To calculate the electronic density of states and the conductance of infinite pristine and functionalized cubane-based chains we have applied the Landauer-Büttiker formalism [3336]. The general illustration of our scheme is presented in Figure 2.

The device region (D) consisting of three substituted cubylene units is connected to left $(\mathrm{L})$ and right $(\mathrm{R})$ semiinfinite leads. The device area is described by the Hamiltonian $H_{\mathrm{D}}$ and corresponding overlap matrix $S_{\mathrm{D}}$. The conductance at 
TABLE 1: HOMO-LUMO gaps $\Delta_{\mathrm{HL}}$ for various cubane-based oligomers constructed from two and four fragments.

\begin{tabular}{lcccc}
\hline \multicolumn{4}{c}{ Radical } & \multicolumn{3}{c}{$\Delta_{\mathrm{HL}}(\mathrm{eV})$} \\
& $\mathrm{N}$ Dimers & \multicolumn{2}{c}{ Tetramers } \\
DFT/PBE/ & NTBM & $\begin{array}{c}\text { DFT/PBE/ } \\
6-31+\mathrm{G}(\mathrm{d}, \mathrm{p})\end{array}$ \\
\hline $\mathrm{H}$ (pristine chain) & 5.17 & 5.31 & 4.91 & 5.02 \\
$\mathrm{CH}_{3}$ & 5.05 & 5.06 & 4.80 & 4.81 \\
$\mathrm{OH}$ & 4.59 & 4.34 & 4.38 & 4.13 \\
$\mathrm{NH}_{2}$ & 4.49 & 3.92 & 4.27 & 3.73 \\
$\mathrm{CN}$ & 3.40 & 5.21 & 3.33 & 4.97 \\
$\mathrm{COOH}$ & 3.01 & 3.64 & 2.98 & 3.62 \\
$\mathrm{NO}_{2}$ & 2.27 & 3.20 & 2.25 & 3.06 \\
$\mathrm{NO}$ & 0.60 & 1.17 & 0.60 & 1.08
\end{tabular}

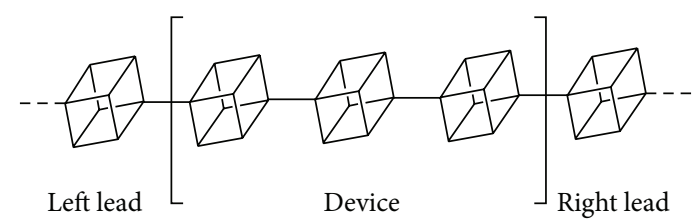

FIGURE 2: General scheme of the considered samples comprising three parts: semi-infinite left and right leads and device area, consisting of three substituted cubylene fragments, sandwiched between them.

zero temperature and in low bias limit can be obtained from Landauer's theory for transport

$$
G(E)=G_{0} \operatorname{Tr}\left(\Gamma_{\mathrm{L}} \mathscr{G} \Gamma_{\mathrm{R}} \mathscr{G}^{\dagger}\right),
$$

where $G$ is the conductance expressed within Fisher-Lee relationship [34], while $G_{0}=2 e^{2} / h$ is the conductance quantum, $E$ is the energy of the incident charge carrier, and $\mathrm{Tr}$ denotes the trace of corresponding operator; $\mathscr{G}$ is the retarded Green's function of the functionalized linear cubane-based chain and matrices $\Gamma_{\mathrm{L}, \mathrm{R}}=i\left(\Sigma_{\mathrm{L}, \mathrm{R}}-\Sigma_{\mathrm{R}, \mathrm{L}}^{\dagger}\right)$ consider the coupling to the right or left leads through their self-energies $\Sigma_{\mathrm{L}, \mathrm{R}}$. The self-energies of the device-leads system can be described as

$$
\begin{aligned}
& \Sigma_{\mathrm{L}}= {\left[(E+i \eta) S_{\mathrm{LD}}-H_{\mathrm{LD}}\right]^{\dagger} } \\
& \cdot\left\{(E+i \eta) S_{\mathrm{L}}^{0}-H_{\mathrm{L}}^{0}+\left((E+i \eta) S_{\mathrm{L}}^{01}-H_{\mathrm{L}}^{01}\right)^{\dagger} \bar{T}_{\mathrm{L}}\right\}^{-1} \\
& \cdot {\left[(E+i \eta) S_{\mathrm{LD}}-H_{\mathrm{LD}}\right] ; } \\
& \Sigma_{\mathrm{R}}= {\left[(E+i \eta) S_{\mathrm{DR}}-H_{\mathrm{DR}}\right] } \\
& \cdot\left\{(E+i \eta) S_{\mathrm{R}}^{0}-H_{\mathrm{R}}^{0}+\left((E+i \eta) S_{\mathrm{R}}^{01}-H_{\mathrm{R}}^{01}\right)^{\dagger} T_{\mathrm{R}}\right\}^{-1} \\
& \cdot\left[(E+i \eta) S_{\mathrm{DR}}-H_{\mathrm{DR}}\right]^{\dagger},
\end{aligned}
$$

where $\eta$ is an arbitrary small quantity; $H_{\mathrm{LD}}, H_{\mathrm{DR}}, S_{\mathrm{LD}}$, and $S_{\mathrm{DR}}$ are the coupling Hamiltonians and corresponding overlap matrices describing connections device left lead and device right lead; $H_{\mathrm{L}}^{0}, H_{\mathrm{R}}^{0}, H_{\mathrm{L}}^{01}, H_{\mathrm{R}}^{01}, S_{\mathrm{L}}^{0}, S_{\mathrm{R}}^{0}, S_{\mathrm{L}}^{01}$, and $S_{\mathrm{R}}^{01}$ describe the left/right lead unit cell and coupling between the neighboring unit cells of the lead; $\bar{T}_{\mathrm{L}}$ and $T_{\mathrm{R}}$ are the lead transfer matrices that can be easily computed from $H_{\mathrm{L}}^{0}, H_{\mathrm{L}}^{01}, S_{\mathrm{L}}^{0}, S_{\mathrm{L}}^{01}$ and $H_{\mathrm{R}}^{0}, H_{\mathrm{R}}^{01}, S_{\mathrm{R}}^{0}, S_{\mathrm{R}}^{01}$ via an iterative procedure (see [37-40] for details). Note that in our case left and right leads as well as device region have the same molecular structure. Therefore $H_{\mathrm{L}}^{0} \equiv H_{\mathrm{R}}^{0} \equiv H_{\mathrm{D}}, S_{\mathrm{L}}^{0} \equiv S_{\mathrm{R}}^{0} \equiv S_{\mathrm{D}}$, and $H_{\mathrm{L}}^{01} \equiv H_{\mathrm{R}}^{01} \equiv H_{\mathrm{LD}} \equiv H_{\mathrm{DR}}$, $S_{\mathrm{L}}^{01} \equiv S_{\mathrm{R}}^{01} \equiv S_{\mathrm{LD}} \equiv S_{\mathrm{DR}}$. The retarded Green's function is given by the expression

$$
\mathscr{G}(E)=\left[(E+i \eta) S_{\mathrm{D}}-H_{\mathrm{D}}-\Sigma_{\mathrm{L}}-\Sigma_{\mathrm{R}}\right]^{-1} .
$$

The electronic density of states (DOS) is given by

$$
\operatorname{DOS}(E)=\left(-\frac{1}{2 \pi}\right) \operatorname{Tr}\left\{\left(\mathscr{G}-\mathscr{G}^{\dagger}\right) S_{\mathrm{D}}\right\}
$$

The electronic Hamiltonians are calculated from overlap matrix elements using extended Hückel approximation [41] with Anderson distant dependence for Wolfsberg-Helmholtz parameter [42]. The overlap integrals are calculated using standard Slater-Koster-Roothaan procedure [43, 44]. Therefore, the analytical expressions for the overlap integrals greatly simplify the calculation of Hamiltonian. The abovementioned fact as well as good accuracy of NTBM model make this approach very efficient for recursive Green's function method as described in [39].

\section{Results and Discussion}

At first we focus on the case of finite linear cubane-based chains (oligomers) constructed from eleven substituted cubylene fragments. In each fragment only one hydrogen atom is replaced by the following functional groups or radicals: $\mathrm{OH}, \mathrm{CN}, \mathrm{NO}, \mathrm{NH}_{2}, \mathrm{NO}_{2}, \mathrm{CH}_{3}, \mathrm{COOH}, \mathrm{N}, \mathrm{O}, \mathrm{CO}$, and $\mathrm{NH}$. Geometry optimization data demonstrate the instability of cubane-based chains with $\mathrm{N}, \mathrm{O}, \mathrm{CO}$, and $\mathrm{NH}$ groups. Only univalent radicals conserve the cubic identity of the elementary units. Thus, polyvalent radicals mentioned above will not be further considered here. We calculated binding energies $E_{b}$ and HOMO-LUMO gaps $\Delta_{\mathrm{HL}}$ of the remaining chains. The binding energy $E_{b}$ of the chain per atom is determined by the equation

$$
\begin{aligned}
E_{b} & {[\mathrm{eV} / \text { atom }]=\frac{1}{N_{\mathrm{at}}}\{k E(\mathrm{H})+l E(\mathrm{C})+m E(\mathrm{~N})} \\
& \left.+n E(\mathrm{O})-E_{\text {tot }}(\text { chain })\right\},
\end{aligned}
$$

where $N_{\text {at }}=k+l+m+n$ is the total number of atoms in the chain, $E_{\text {tot }}$ (chain) is the total chain (oligomer) energy, $E(\mathrm{H}), E(\mathrm{C}), E(\mathrm{~N})$, and $E(\mathrm{O})$ are the energies of the isolated hydrogen, carbon, nitrogen, and oxygen atoms, respectively. As evident from Table $2 \Delta_{\mathrm{HL}}$ is very sensitive to functional group attached. The narrowest gap is observed for NO-doping (Figure $1(\mathrm{c})$ ); it is equal to $0.60 \mathrm{eV}$. Note that HOMO-LUMO gap is not directly dependent on the binding energy (see Table 2). Therefore, it seems possible to adjust 
TABLE 2: Binding energies $E_{b}$ and HOMO-LUMO gaps $\Delta_{\mathrm{HL}}$ for various cubane-based oligomers obtained within NTBM model.

\begin{tabular}{lcc}
\hline Radical & $\Delta_{\mathrm{HL}}(\mathrm{eV})$ & $E_{b}(\mathrm{eV} /$ atom $)$ \\
\hline $\mathrm{H}$ (pristine chain) & 4.82 & 4.69 \\
$\mathrm{CH}_{3}$ & 4.72 & 4.59 \\
$\mathrm{OH}$ & 4.30 & 4.65 \\
$\mathrm{NH}_{2}$ & 4.19 & 4.54 \\
$\mathrm{CN}$ & 3.30 & 4.96 \\
$\mathrm{COOH}$ & 3.04 & 4.77 \\
$\mathrm{NO}_{2}$ & 2.25 & 4.68 \\
$\mathrm{NO}$ & 0.60 & 4.69 \\
\hline
\end{tabular}

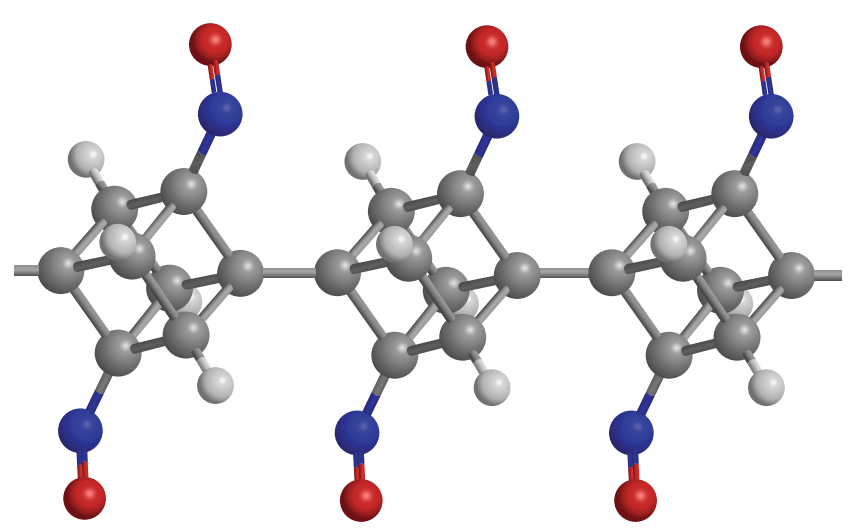

FIgURE 3: Fragment of the double NO-functionalized cubane-based chain.

the electronic structure in a wide range by radical substitution of hydrogen from cubylene unit without loss of thermodynamic stability of the samples.

To study the doping effect in more detail we replace the second hydrogen atom in each elementary unit of NOdoped chain by another NO-group (see Figure 3). As a result $\Delta_{\mathrm{HL}}$ slightly decreases from 0.60 to $0.51 \mathrm{eV}$. Note that the subsequent increase of the number of NO-groups in the chain leads to the stability loss of cubic framework. Probably it may be concerned with the adjacency of functional groups attached. Earlier we found that their "effective repulsion" makes the synthesis of isolated cubanes with adjacent nitrogen-containing groups quite difficult [20]. Thus, we were unable to make the HOMO-LUMO gap disappear and convert the cubane-based chain to metallic state. However, we assume the existence of such combination of radicals (not only $\mathrm{H}-\mathrm{C}-\mathrm{N}-\mathrm{O}$ ) which can close the HOMO-LUMO gap in electronic structure of cubane-based oligomers.

We further explore the conduction regime in chemically functionalized infinite cubane-based chains by calculating the conductance for chains with varying radicals attached. The dependence of the functionalized chain conductance as well as DOS as a function of the radical attached is illustrated in Figure 4 for a set of typical configurations. It is shown that the functionalization alters the electronic properties of the chain since the dopants states are located near the Fermi level.
So, the pristine cubane-based chain is an insulator, while NOgroup substituted chain is a narrow-gap semiconductor.

Note that energy gaps for infinite substituted cubanebased chains are in good agreement with the HOMOLUMO gaps of corresponding eleven-fragment samples. Our numerical calculations show that in pristine cubane-based chain HOMO-LUMO excitation was characterized by electronic density transfer to the ends of chain. By contrast, in narrow-gap substituted cubane-based chains (e.g., NOfunctionalized chain) electronic density rearranges to the functional groups. So, the fine adjustment of HOMO-LUMO gap has its origins in electronic density transfer to the corresponding radicals. Therefore the HOMO-LUMO gap depends on the functional groups selected. The important consequence is that the functionalization of the cubane-based chain leads to the opening of new conduction channels (see Figure 4): for functionalized chains the energy gap becomes narrower since the dopants states are located near the Fermi level. Therefore, they exhibit semiconducting properties, and conductivity becomes possible at nonzero temperatures. This fact indicates that the functionalized cubane-based chains can be potentially used as effective conductors in nanodevices.

\section{Conclusions}

We have studied electronic properties of the cubane-based compounds containing different chemical groups with various electronegativities. It is shown that such type of functionalization alters the electronic properties of the chain since the dopants states are located near the Fermi level. Some functional groups or radicals can significantly reduce the energy gap by opening the additional conduction channels in the molecular structure. Moreover, the increase of the number of chemical groups (e.g., NO-groups) leads to further decreasing the gap, but on the other hand it may lead to the stability loss and decomposition of the chain. So, we were unable to convert the cubane-based chain to metallic state by removing the hydrogen and adding the functional group instead of it. Maybe it is possible to make the gap disappear by implementing the additional "molecular bridges" between the substituted cubylene fragments of the cubane-based compound. Anyway it has become possible to tune electronic properties of the cubane-based chains by the chemical functionalization that will be useful for nanoelectronic applications.

\section{Conflict of Interests}

The authors declare that there is no conflict of interests regarding the publication of this paper.

\section{Acknowledgments}

This work was supported by the Russian Foundation for Basic Research, Project no. 14-02-31416-mol_a. The useful comments of anonymous reviewers are gratefully acknowledged. 

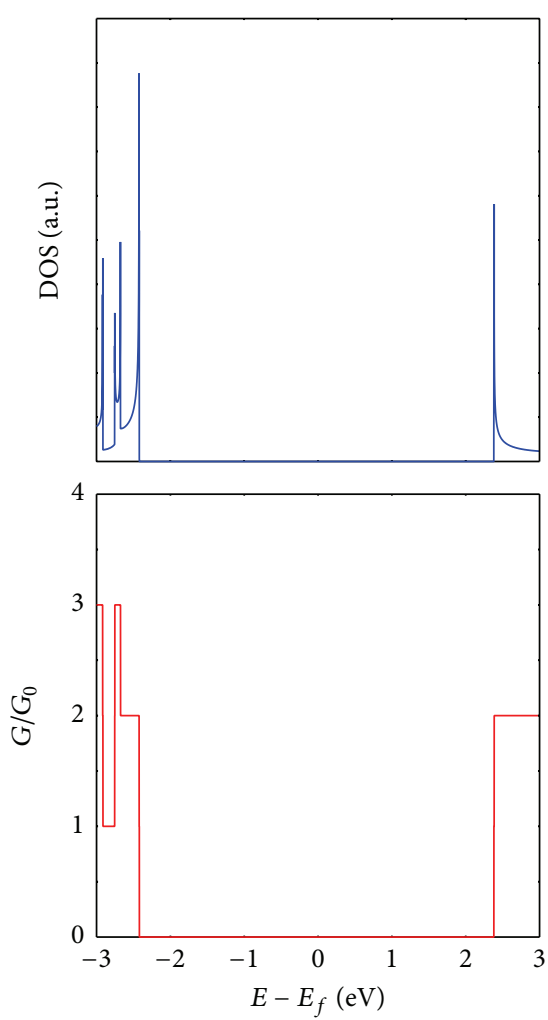

(a)
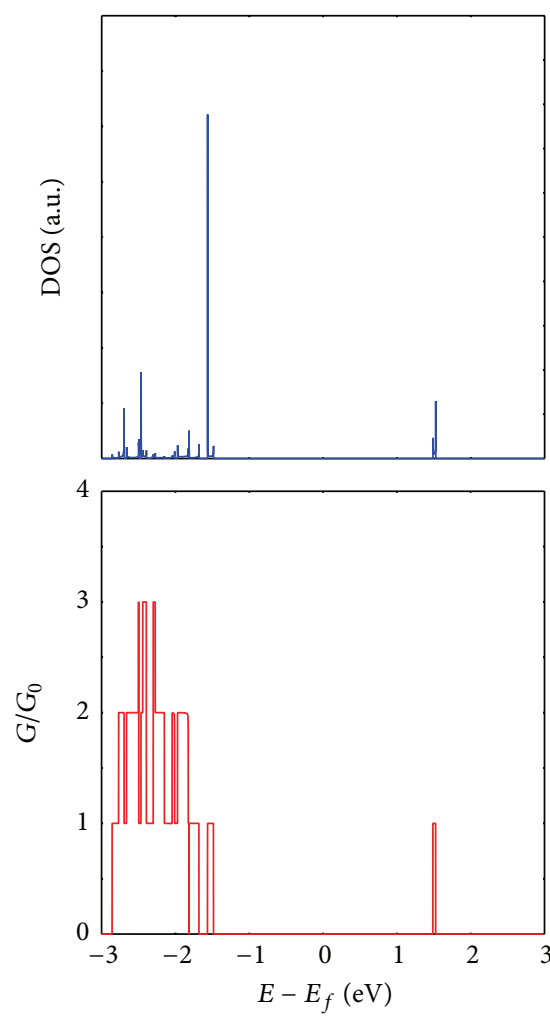

(b)
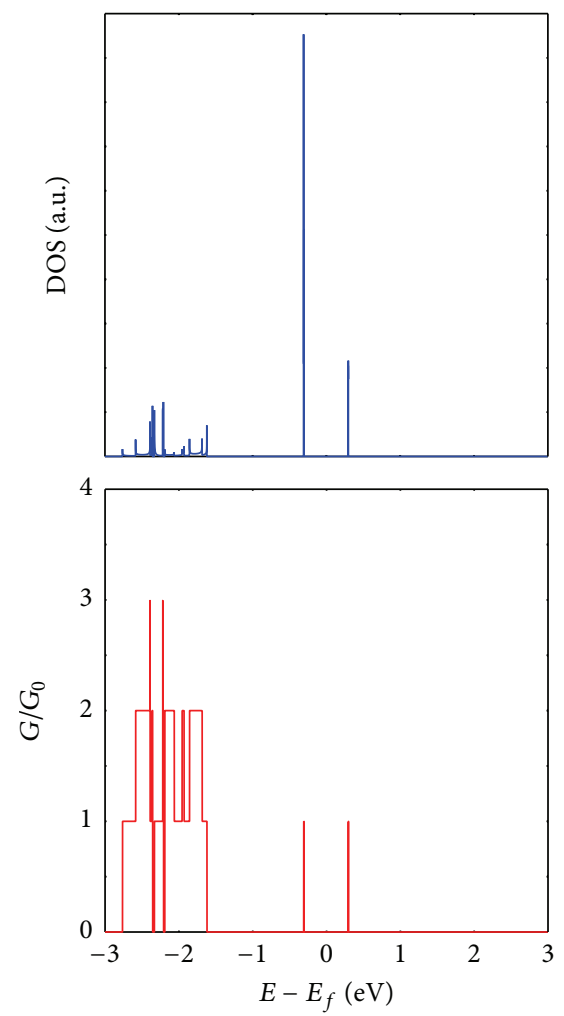

(c)

FIGURE 4: Density of electronic states (blue) and conductance (red, in units of $G_{0}=2 e^{2} / h$ ) of the infinite cubane-based chains for the pristine case (a) and for the $\mathrm{COOH}$ - and $\mathrm{NO}$-functionalized cases ((b) and (c), resp.).

\section{References}

[1] P. E. Eaton and T. W. Cole Jr., “The cubane system,” Journal of the American Chemical Society, vol. 86, no. 5, pp. 962-964, 1964.

[2] F. Agapito, R. C. Santos, R. M. Borges dos Santos, and J. A. Martinho Simões, "The thermochemistry of cubane 50 years after its synthesis: a high-level theoretical study of cubane and its derivatives," The Journal of Physical Chemistry A, vol. 119, no. 12, pp. 2998-3007, 2015.

[3] M. M. Maslov, D. A. Lobanov, A. I. Podlivaev, and L. A. Openov, "Thermal stability of cubane $\mathrm{C}_{8} \mathrm{H}_{8}$," Physics of the Solid State, vol. 51, no. 3, pp. 645-648, 2009.

[4] P. E. Eaton, J. Li, and S. P. Upadhyaya, "Synthesis of methylcubane and cyclopropylcubane. The cubane-1,4-diyl route," The Journal of Organic Chemistry, vol. 60, no. 4, pp. 966-968, 1995.

[5] P. E. Eaton, K. Pramod, T. Emrick, and R. Gilardi, "Building with cubane-1,4-diyl. Synthesis of aryl-substituted cubanes, $p$ [n]cubyls, and cubane-separated bis(arenes)," Journal of the American Chemical Society, vol. 121, no. 17, pp. 4111-4123, 1999.

[6] P. E. Eaton, R. L. Gilardi, and M.-X. Zhang, "Polynitrocubanes: advanced high-density, high-energy materials," Advanced Materials, vol. 12, no. 15, pp. 1143-1148, 2000.

[7] M. X. Zhang, P. E. Eaton, and R. L. Gilardi, "Hepta- and octanitrocubanes," Angewandte Chemie International Edition, vol. 39, no. 2, pp. 401-404, 2000.

[8] P. E. Eaton, M.-X. Zhang, R. L. Gilardi, N. Gelber, S. Iyer, and R. Surapaneni, "Octanitrocubane: a new nitrocarbon," Propellants, Explosives, Pyrotechnics, vol. 27, no. 1, pp. 1-6, 2002.
[9] R. Gilardi, M. Maggini, and P. E. Eaton, "X-ray structures of cubylcubane and 2-tert-butylcubylcubane: short cage-cage bonds," Journal of the American Chemical Society, vol. 110, no. 21, pp. 7232-7234, 1988.

[10] T. Yildirim, "Structure and dynamics from combined neutron scattering and first-principles studies," Chemical Physics, vol. 261, no. 1-2, pp. 205-216, 2000.

[11] T. Yildirim, S. Ciraci, Ç. Kiliç, and A. Buldum, "First-principles investigation of structural and electronic properties of solid cubane and its doped derivatives," Physical Review B, vol. 62, no. 11, pp. 7625-7633, 2000.

[12] F. Valencia, A. H. Romero, M. Kiwi, R. Ramírez, and A. ToroLabbe, "Ab initio study of cubyl chains and networks," The Journal of Chemical Physics, vol. 121, no. 18, pp. 9172-9177, 2004.

[13] B. Herrera, F. Valencia, A. H. Romero, M. Kiwi, R. Ramírez, and A. Toro-Labbé, "Cubane oligomers: a density functional theory study," Journal of Molecular Structure: THEOCHEM, vol. 769, no. 1-3, pp. 183-187, 2006.

[14] M. M. Maslov, "Theoretical modeling of the cubane-based chains, networks and bulks," Journal of Physics: Conference Series, vol. 129, Article ID 012045, 2008.

[15] M. M. Maslov, K. P. Katin, A. I. Avkhadieva, and A. I. Podlivaev, "Dynamic modeling of cyclotetracubyl thermal decomposition," Russian Journal of Physical Chemistry B, vol. 8, no. 2, pp. 152-157, 2014.

[16] E. Konstantinova, A. Camilo Jr., P. M. V. B. Barone, S. O. Dantas, and D. S. Galvão, "Some electronic properties of saturated and unsaturated cubane oligomers using DFT-based calculations," 
Journal of Molecular Structure: THEOCHEM, vol. 868, no. 1-3, pp. 37-41, 2008.

[17] F. Valencia, A. H. Romero, M. Kiwi, R. Ramírez, and A. ToroLabbe, "Polycubanes linked with $\mathrm{C}_{2}, \mathrm{~N}_{2}, \mathrm{NO}$, and NS: from insulating to metallic behavior," Physical Review B, vol. 71, no. 3, Article ID 033410, 2005.

[18] E. Konstantinova, P. M. V. B. Barone, and S. O. Dantas, "On the dependence of electronic properties on geometric structure for cubane derivatives and oligomers with donor and acceptor groups," Journal of Molecular Structure: THEOCHEM, vol. 909, no. 1-3, pp. 116-121, 2009.

[19] P. P. Pal and R. Pati, "Charge transport in strongly coupled molecular junctions: 'in-phase' and 'out-of-Phase' contribution to electron tunneling," The Journal of Physical Chemistry C, vol. 115, no. 35, pp. 17564-17573, 2011.

[20] K. P. Katin and M. M. Maslov, "Thermal stability of nitro derivatives of hydrocarbon cubane," Russian Journal of Physical Chemistry B, vol. 5, no. 5, pp. 770-779, 2011.

[21] Nonorthogonal Tight-Binding Model version number 1, http://ntbm.info/.

[22] C. Lee, W. Yang, and R. G. Parr, "Development of the ColleSalvetti correlation-energy formula into a functional of the electron density," Physical Review B, vol. 37, no. 2, pp. 785-789, 1988.

[23] A. D. Becke, "Density-functional thermochemistry. III. The role of exact exchange," The Journal of Chemical Physics, vol. 98, no. 7, article 5648, 1993.

[24] J. P. Perdew, K. Burke, and M. Ernzerhof, "Generalized gradient approximation made simple," Physical Review Letters, vol. 77, no. 18 , pp. 3865-3868, 1996.

[25] R. Krishnan, J. S. Binkley, R. Seeger, and J. A. Pople, "Selfconsistent molecular orbital methods. XX. A basis set for correlated wave functions," The Journal of Chemical Physics, vol. 72, no. 1, pp. 650-654, 1980.

[26] M. W. Schmidt, K. K. Baldridge, J. A. Boatz et al., "General atomic and molecular electronic structure system," Journal of Computational Chemistry, vol. 14, no. 11, pp. 1347-1363, 1993.

[27] M. M. Maslov, A. I. Podlivaev, and K. P. Katin, "Nonorthogonal tight-binding model with $\mathrm{H}-\mathrm{C}-\mathrm{N}-\mathrm{O}$ parameterisation," Molecular Simulation, 2015.

[28] NIST Standard Reference Database 101, Computational Chemistry Comparison and Benchmark DataBase, http://cccbdb.nist .gov/.

[29] M. B. Javan, "Electronic transport properties of linear $n \mathrm{C}_{20}(n \leq 5)$ oligomers: theoretical investigation," Physica E: Low-Dimensional Systems and Nanostructures, vol. 67, pp. 135-142, 2015.

[30] N. Ghobadi and Y. Abdi, "Device characteristics and tight binding based modeling of bilayer graphene field-effect transistor," Current Applied Physics, vol. 13, no. 6, pp. 1082-1089, 2013.

[31] H. Rabani, M. Mardaani, and A. M. Shahraki, "Analytical study of the ballistic transport of ladder-like graphene nanoribbons within the tight-binding approach," Superlattices and Microstructures, vol. 59, pp. 106-114, 2013.

[32] W. Sukkabot, "Structural properties of $\mathrm{SiC}$ zinc-blende and wurtzite nanostructures: atomistic tight-binding theory," Materials Science in Semiconductor Processing, vol. 40, pp. 117-122, 2015.

[33] R. Landauer, "Electrical resistance of disordered one-dimensional lattices," Philosophical Magazine, vol. 21, no. 172, pp. 863867, 1970.
[34] D. S. Fisher and P. A. Lee, "Relation between conductivity and transmission matrix," Physical Review B, vol. 23, no. 12, article 6851, 1981.

[35] M. Büttiker, Y. Imry, R. Landauer, and S. Pinhas, "Generalized many-channel conductance formula with application to small rings," Physical Review B, vol. 31, no. 10, pp. 6207-6215, 1985.

[36] M. Büttiker, "Four-terminal phase-coherent conductance," Physical Review Letters, vol. 57, no. 14, pp. 1761-1764, 1986.

[37] M. P. L. Sancho, J. M. L. Sancho, and J. Rubio, "Quick iterative scheme for the calculation of transfer matrices: application to Mo (100)," Journal of Physics F: Metal Physics, vol. 14, no. 5, pp. 1205-1215, 1984.

[38] M. P. L. Sancho, J. M. L. Sancho, and J. Rubio, "Highly convergent schemes for the calculation of bulk and surface Green functions," Journal of Physics F: Metal Physics, vol. 15, no. 4, article 851, 1985.

[39] M. B. Nardelli, "Electronic transport in extended systems: application to carbon nanotubes," Physical Review B, vol. 60, no. 11, pp. 7828-7833, 1999.

[40] T. Markussen, R. Rurali, M. Brandbyge, and A.-P. Jauho, "Electronic transport through Si nanowires: role of bulk and surface disorder," Physical Review B, vol. 74, no. 24, Article ID 245313, 2006.

[41] R. Hoffmann, "An extended Hückel theory. I. Hydrocarbons," The Journal of Chemical Physics, vol. 39, no. 6, p. 1397, 1963.

[42] A. B. Anderson, "Derivation of the extended Hückel method with corrections: one electron molecular orbital theory for energy level and structure determinations," The Journal of Chemical Physics, vol. 62, no. 3, pp. 1187-1188, 1975.

[43] J. C. Slater and G. F. Koster, "Simplified LCAO method for the periodic potential problem," Physical Review, vol. 94, no. 6, pp. 1498-1524, 1954.

[44] C. C. J. Roothaan, "A study of two-center integrals useful in calculations on molecular structure. I," The Journal of Chemical Physics, vol. 19, pp. 1445-1458, 1951. 

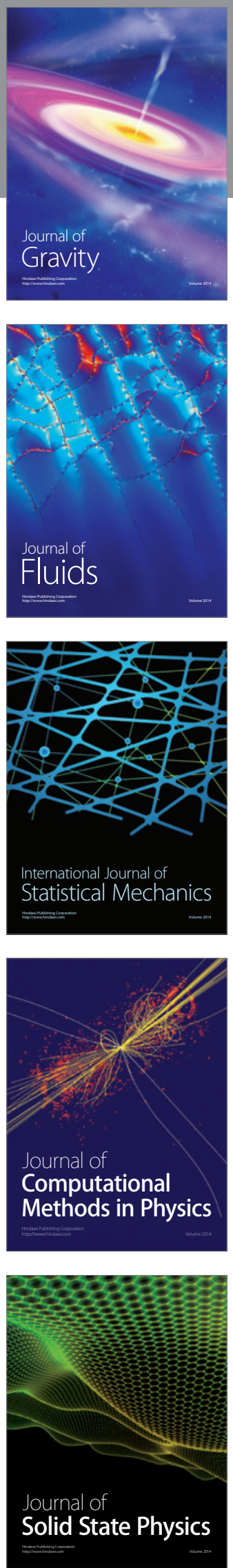

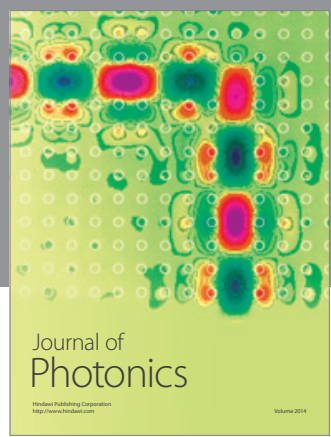

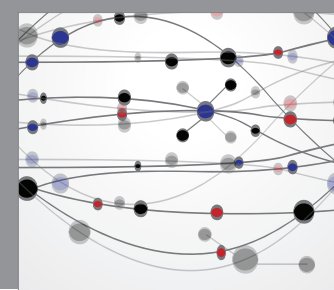

The Scientific World Journal

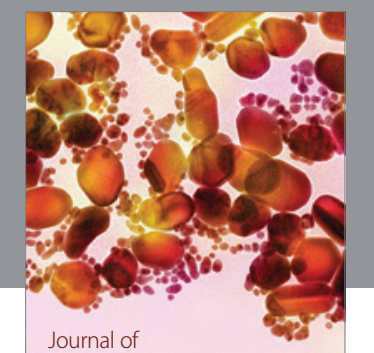

Soft Matter
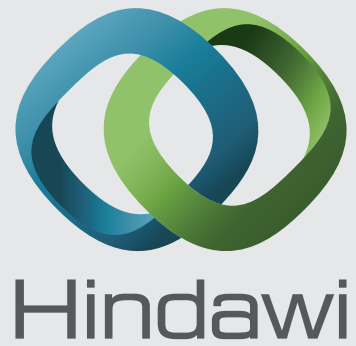

Submit your manuscripts at

http://www.hindawi.com
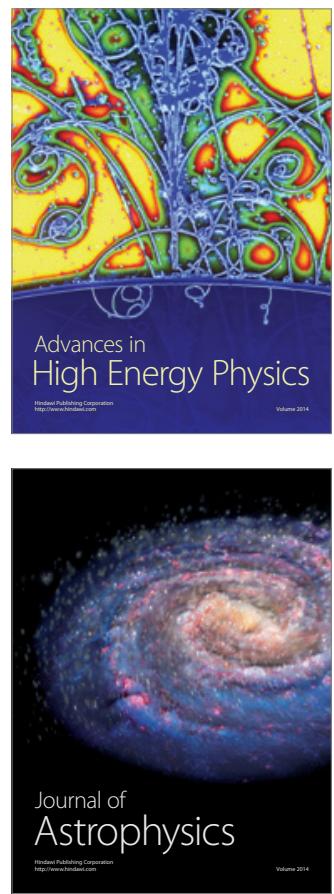
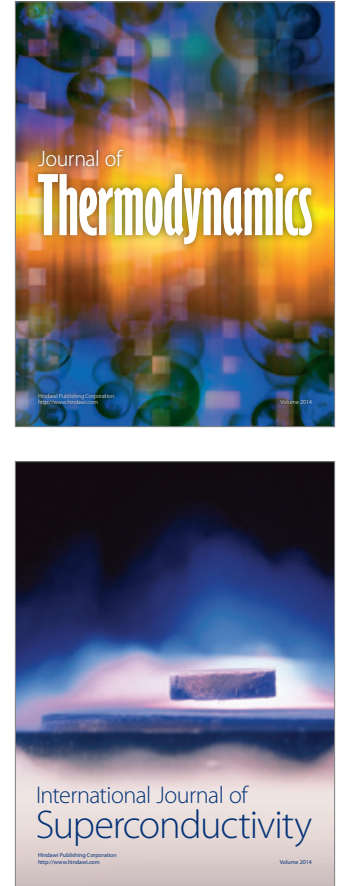
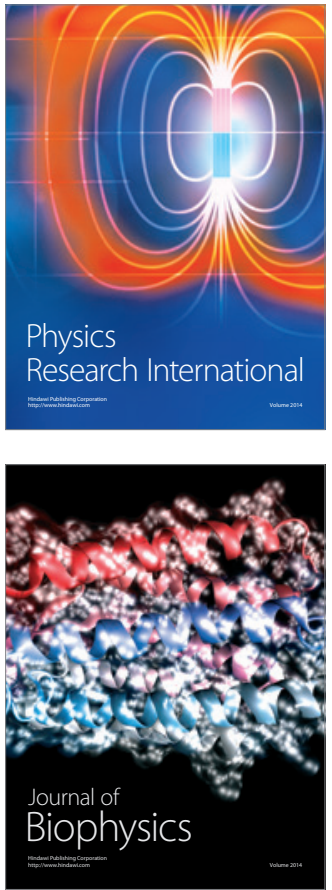
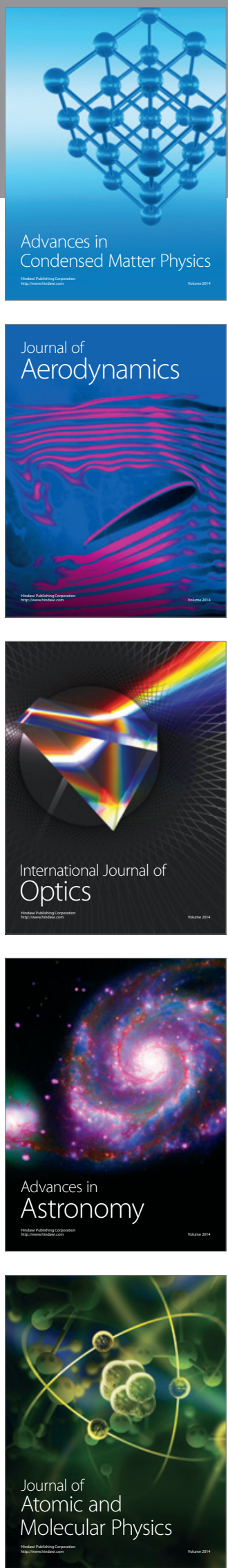